\title{
IS A PICTURE WORTH A THOUSAND WORDS? THE USE OF NON-TEXT ITEMS IN INFORMATION SYSTEMS RESEARCH.
}

\author{
Alison Parkes \\ Department of Information Systems \\ Massey University \\ Private Bag 11222 \\ Palmerston North \\ New Zealand \\ a.j.parkes@massey.ac.nz
}

\begin{abstract}
The use of non-text items (graphs, graphics and tables) is firmly established in the publishing tradition of information systems research. Non-text images are used to convey complex information in a pictorial or diagrammatic sense, adding additional meaning to the discussion. Non-text images also highlight results, and meaningfully represent both quantitative and qualitative data.

This paper examines the use of non-text items in information systems joumals. Three measures of use are employed: rate of use of non-text items; academic and practitioner journal usage of non-text items; and citation rates of articles with varying levels of non-text items. The sample research articles represent a 30year timeframe and include articles from six prominent information systems journals. The research results show an increasing use of non-text items in information systems research. In addition, articles that utilise a higher rate of non-text items were more likely to be cited in other works. Practitioner journals were found to utilise more non-text items than academic journals, and to utilise a different mixture of non-text items.

It would appear that articles that use higher levels of non-text items are more widely cited, so if citedness is the definition of 'success', then it may be practical to incorporate a range of non-text items. If consideration is given to the motive behind selection of articles for citation when preparing research, it seems possible that increased use of non-text items improves the clarity of research findings, leading to their increased usage, uptake and citation levels.
\end{abstract}

\section{INTRODUCTION}

Information accumulated during research endeavours can be presented in a variety of ways. The most common methods are via text, (or narrative), or by non-text items depicting the information. There is minimal published research available in this area however a summary of some findings to date follows.

An examination of how user perceptions and decision making varied under three different methods of information presentation (tabular, graphical and combined tabular graphical) found that when time limits were low, tabular reports produced better decisions, whereas graphic reports produced quicker decisions. Combined tabular and graphic representations produced both faster and better decision making, and was found to be the preferred report format for most decision makers. Benbasat \& Dexter. (1986).

Readers tend to assign higher levels of trust in data presented in a scientific manner. It was found that using numerical tables tended to make readers assume the existence of scientific rigor. Davis (1981). Information presented in a combination of text and non-text styles is more likely to be comprehended by the recipient of the information presentation. Hamilton \& Ives (1983). The increasing use of graphics in technical writing may be related to the emergence of technology that facilitates usage of graphics in both oral and written information presentations. Owen \& van Halm (1989).

Advances in publishing technology are likely to have facilitated increased usage of non-text items, however slow uptake of new technology may act as an obstacle to innovation in the publishing industry. McGill (1990).

Research on the impact of various combinations of format and summarisation levels on different personality types defined by the Myers-Briggs typing instrument found that where the user personality type is unknown, raw data is the best offering, whereas if the personality type is known, there is a preferred option for each cognitive style. It is interesting to note that perceptions of value of information will vary between different personality types. Moran \& Journet (1985).

\section{Research Question}

The purpose of this research is to determine whether the use of non-text items is increasing in published research, whether the use of non-text items affects the level of citedness of articles, and if there are 
differing levels of usage of non-text items between practitioner and academic journals. Three hypothesis have been formulated.

H1 That the rate of occurrence of non-textual information in published Information systems research is increasing over time.

Because of increasing awareness of the value of non-text items, and the improved ability of publishers to present non-text items easily, it was theorised that research would increasingly use non-text representations of data

\section{H2 That practitioner journals would exhibit higher frequency of non-text occurrences than} academic journals.

There are two main types of journals where information systems research is published, categorised as academic and practitioner journals. Practitioner journals tend to be 'glossy', with a magazine style format. It was thought that due to the form of the journal, and the target audience, practitioner journals would use more non-text representations than academic journals.

\section{H3 That those articles that contain a higher number of occurrences of non-textual data are more often cited.}

The existence of higher numbers of non-text items in an article increases readability and comprehension of the content. It is thought that successful information systems research would use higher levels of nontext information. Those articles that contain a high number of non-text representations may be more widely used, and, if so, would be cited more frequently.

To test these hypotheses, a representative sample of journals was selected for examination. As an aid to determining a representative sample, a typology of journals was used to guide the selection. Vogel \& Wetherbe (1984). A representative sample of articles was examined and occurrences of non-textual data recorded.

\section{Definition of Non-text items}

For the purposes of this research the following definitions apply:

Table: Information displayed in matrix format, which is labelled or identified as a Table within the article e.g.

\begin{tabular}{|c|c|c|}
\hline \multicolumn{3}{|c|}{ 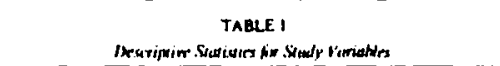 } \\
\hline & mean & Sed dev. \\
\hline Responsibility & 0.29 & $0.3 !$ \\
\hline lisnderm Artivioiss & 0.42 & 0.32 \\
\hline User-15 Retationstip & 0.38 & 0.30 \\
\hline Influense-item ! & .31 & 2.20 \\
\hline In luevere-itern 2 & 4.97 & 1.9 \\
\hline Inferense-isema 3 & 0.49 & 206 \\
\hline Confiet-isem I & 2.88 & 1.76 \\
\hline Cimanx-iten 2 & 3.46 & 2.11 \\
\hline Connikt-ikem 3 & 367 & 1.93 \\
\hline Sanifiationy Connlist Reedutution-ikm I & .83 & 1.82 \\
\hline 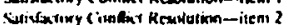 & $.7 \pi$ & 1.79 \\
\hline
\end{tabular}

Figure 1. Example of Table 


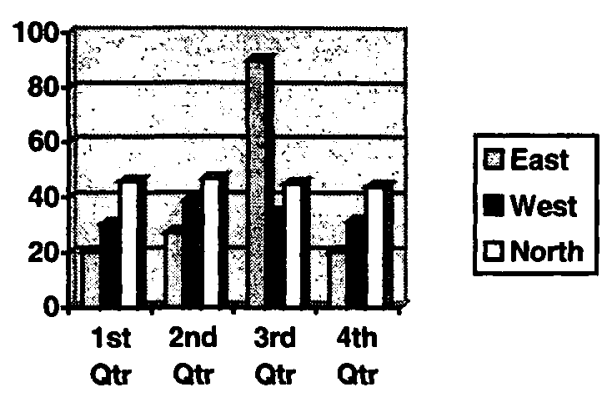

Figure 2. Example of Graph

Graph: A pictorial representation of quantitative data made in a non-numeric illustration, for example a bar chart or pie graph. e.g.

GraphicA picture or model illustration e.g.

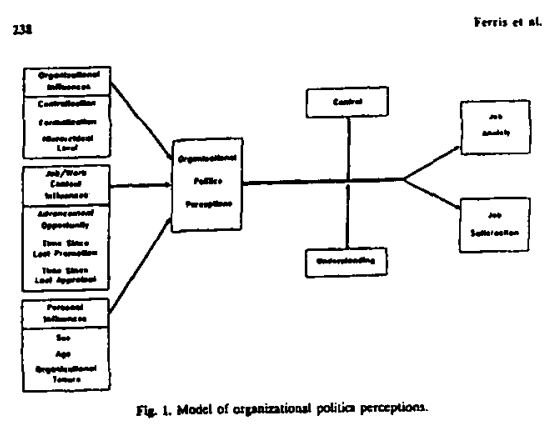

Figure 3. Example of Graphic

METHODS

\section{Selection of Journals}

Journals were stratified into four levels of importance, and those levels divided into practitioner and academic orientations. Two journals were selected from each orientation for level 1 , and one journal was selected from each orientation for level 2 of the taxonomy; a total of six journals. Levels 3 and 4 of the taxonomy were not considered as the information provided was too generalised to aid in journal selection.

In order to select the most appropriate journals to utilise within each classification, the citation frequency was utilised. The citation frequency was combined with the corresponding taxonomy of journal importance. Journals that were identified as attaining a higher number of citations within each level, for each orientation, were selected for this research. The rationale behind the utilisation of the citation frequency as the selector is that testing $\mathrm{H} 3$ will involve the use of a citation index. Sample articles are more likely to appear in this index if high citation frequency has been advised for their publishing journal.

The most important and frequently cited journals from levels 1 and 2, for both academic and practitioner orientation were as follows: 


\begin{tabular}{|c|c|c|}
\hline Level & Orientation & Selected Journal \\
\hline \multirow{4}{*}{1} & \multirow{2}{*}{ Academic } & Management Science \\
\cline { 2 - 3 } & \multirow{2}{*}{ Practitioner } & MIS Quarterly \\
\cline { 2 - 3 } & Academic & Datamation \\
\hline \multirow{2}{*}{2} & Practitioner & Harvard Business Review \\
\cline { 2 - 3 } & & Communications of the ACM \\
\hline
\end{tabular}

Table 1. Selected Journals

As the same journal was selected for both academic and practitioner orientation for level 2 , it was decided to examine the second most frequently cited journal for those levels, the Journal of Systems Management. To determine which journal was most representative of academic orientation, an analysis of submission preferences was conducted. This showed a higher academic preference for submission to Communications of the ACM. The journals selected for the final sample were:

\begin{tabular}{|c|c|c|}
\hline Level & Orientation & Selected Joarnal \\
\hline \multirow{4}{*}{1} & Academic & Management Science \\
\cline { 2 - 3 } & & MIS Quarterly \\
\cline { 2 - 3 } & \multirow{3}{*}{ Practitioner } & Datamation \\
\cline { 2 - 3 } & & Harvard Business Review \\
& Academic & \\
\hline \multirow{2}{*}{2} & Practitioner & Communications of the ACM \\
\hline
\end{tabular}

Table 2. Final Journal Selection

\section{Selection of Articles}

In order to test $\mathrm{H} 1$ : That the rate of occurrence of non-textual information in published Information systems research is increasing over time, a longitudinal sample of journal articles was required. It was decided to sample from the years $1991,1993,1995$ \& 1997. The first issue for each year of the selected journals was selected. Every article in the selected issues was examined. This resulted in a sample of 201 articles; containing 916 identified non-textual information representations.

Additional articles from 1971 and 1981, selected in the same manner, were added during the research process, to broaden the longitudinal scope of the research. This resulted in a total sample size of 271 articles containing 1148 non-text images.

\section{Selection of Citation Index}

There are two possible information sources relating to citation frequency, the Social Sciences Citation Index and the Science Citation Index. To determine the most appropriate index to use, an examination was conducted of the source listing for each index, with the results shown in table 3: 


\begin{tabular}{|l|c|c|}
\hline \multirow{2}{*}{ Joumal } & Social Science Citation & $\frac{\text { Science Citation Index }}{\text { Index Source }}$ \\
\hline Management Science & $\checkmark$ & $\times$ \\
\hline Datamation & $\times$ & $\times$ \\
\hline Harvard Business Review & $\checkmark$ & $\times$ \\
\hline MIS Quarterly & $\checkmark$ & $\times$ \\
\hline Communications of the ACM & $\checkmark$ & $\times$ \\
\hline Journal of Systems Management & $\times$ & $\times$ \\
\hline
\end{tabular}

Table 3. Citation Index Sources

It was established that the Social Science Citation Index was the most appropriate source to use for checking citation frequency of the selected journal articles.

\section{Selection of Articles for citation checking.}

Articles for citation checking were selected from journals listed in the sources of the Social Science Citation index; viz, Management Science, Harvard Business Review, MIS Quarterly and Communications of the ACM. The articles in these magazines were grouped into low, medium and high usage level groups. Low usage was defined as $0-3$ occurrences, medium as $4-8$ occurrences and high as 9 or more occurrences of non-text items in an individual article. Sample articles were randomly selected from journals in the year 1995. Four articles were selected for each usage level, a total of 12 articles.

\section{RESULTS}

Analysis of data relating to $\mathrm{H} 1$ : That the rate of occurrence of non-textual information in published Information Systems Research is increasing over time

The initial sample, when analysed by calculating the mean of occurrences, resulted in no clearly identifiable trend relating to the increasing use of non-textual items as shown in figure 4.

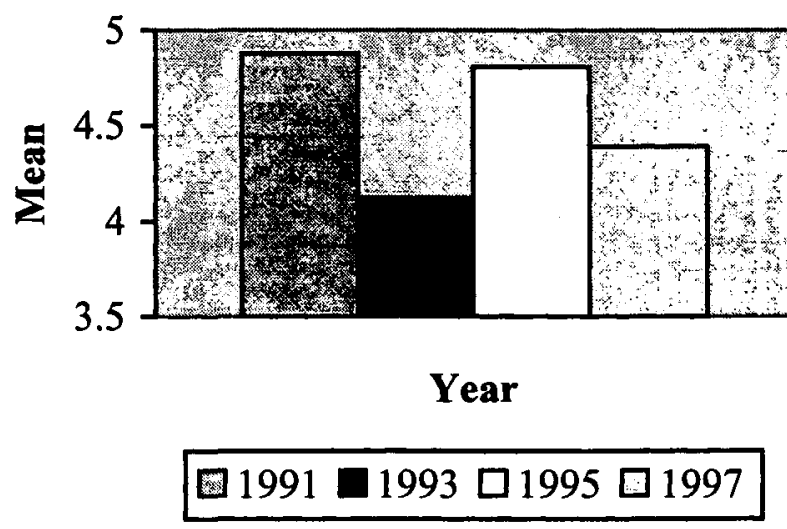

Figure 4. Initial Sample - mean of non-text item occurrence 
To determine if longitudinal magnitude was an issue, a further set of observations was made. Utilising the previously identified journals, a sample was taken of articles from the first edition for each of the years 1971, and 1981. These results were added to material collected for 1991 .

Following the amendment of the sample to constitute 1971, 1981 and 1991, the number of articles examined was as shown in table 4 :

\begin{tabular}{|l|l|c|}
\hline Orientation & \multicolumn{1}{|c|}{ Selected Journal } & Number of Articles \\
\hline \multirow{3}{*}{ Academic } & Management Science & 27 \\
\cline { 2 - 3 } & MIS Quarterly & 9 \\
\cline { 2 - 3 } & Communications of the ACM & 18 \\
\hline \multirow{3}{*}{ Practitioner } & Datamation & 17 \\
\cline { 2 - 3 } & Harvard Business Review & 35 \\
\cline { 2 - 3 } & Journal of Systems Management & 12 \\
\hline \multicolumn{2}{|c|}{ Total } & 118 \\
\hline
\end{tabular}

Table 4. Summary of articles selected

The mean for the first year of each decade was graphed, see figure 5 for the results:

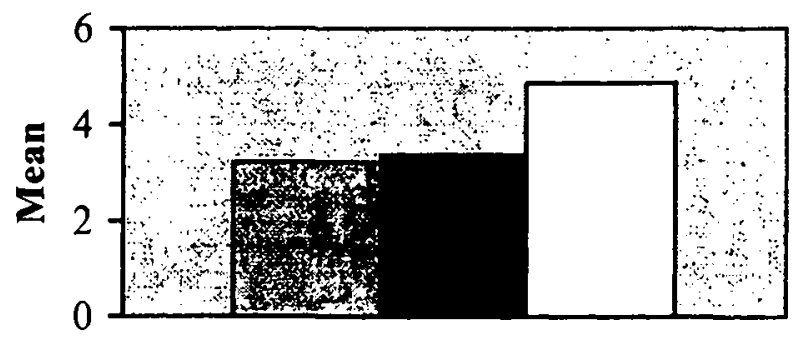

Year

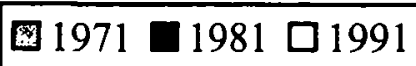

Figure S. Final sample - mean of non-text occurrences by decade

The results showed an increasing rate of usage of non-text items. Therefore $\mathrm{H} 1$ : that the rate of occurrence of non-textual information in published information systems research is increasing over time; was supported.

\section{Analysis of data relating to $\mathrm{H2}$ : that practitioner articles would exhibit higher frequency of non- text occurrences than academic journals.}

In order to test H2: that practitioner journals would exhibit higher frequency of non-text occurrences than academic journals; a comparison was made between the mean of occurrences of non-text items in academic journals and those in practitioner journals. Given a length disparity between academic and practitioner articles (practitioner article average length three pages; academic article average length ten pages) the frequency of usage of non-text items relative to the length of the published article was assessed with results shown in figure 6 . 


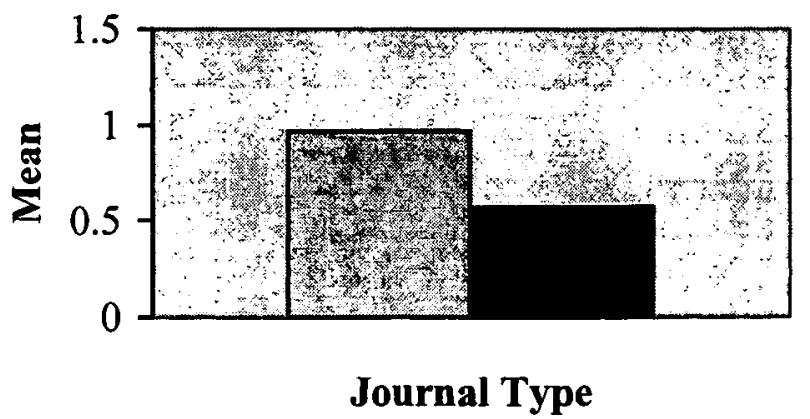

\section{圆Practitioner $\mathbf{D}$ Academic}

Figure 6. Mean of occurrences of non-text information, practitioner and academic journals.

These results support the suggestion that practitioner-oriented journals exhibit higher frequency of nontext occurrences than academic journals. The outcome of the analysis was that practitioner journals in fact use nearly twice as many non-text items as academic journals. An examination of the usage levels of different types of non-text items used in the publications is depicted in figure 7 :

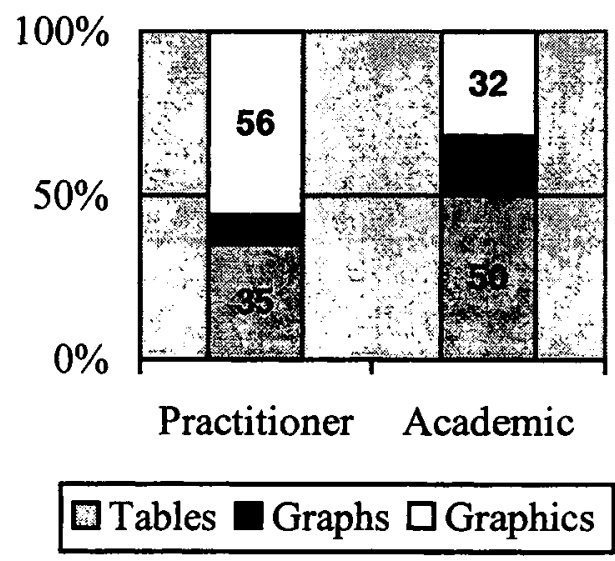

Figure 7. Comparison by percentage of types of non-text items used

These results suggest that articles published in practitioner journals relied heavily on the use of graphics to support text, whereas articles published in academic journals used tabular presentation of material more frequently.

\section{Analysis of data relating to $\mathrm{H3}$ : that those items that contain a higher number of occurrences on non-text data are more often cited.}

The articles selected for citation checking were verified against the Social Science Citation Index for the years 1995, 1996, \& 1997, and for the first quarter of 1998. The results for the identified low, medium and high usage of non-text item usage were as follows in figure 8: 


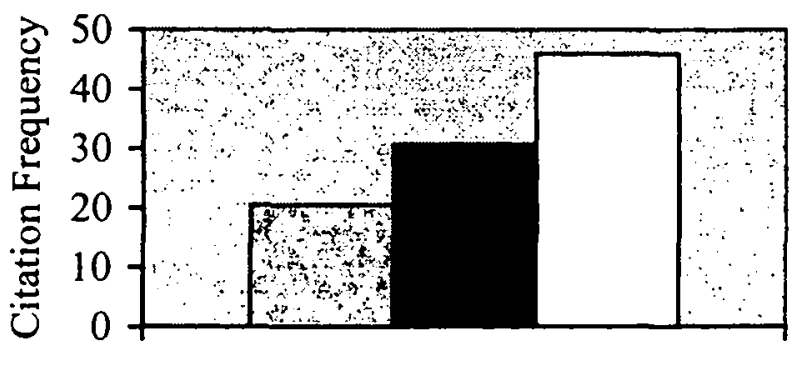

Usage level

\section{国 Low Medium $\square$ High}

Figure 8. Mean of citation frequency

These results support H3: That those articles that contain a higher number of occurrences of non-textual data are more often cited.

\section{CONCLUSION}

The results of the research show that information systems research is using increasing numbers of nontext items in published research. The results also identified that practitioner journals utilise more nontext items, relative to length, than academic journals; and that articles with a higher usage rate of nontext items are more likely to be cited.

These results were accomplished by selecting a sample of articles and analysing the frequency of nontext item occurrences. All analysis was based on comparison of the means of occurrence of non-text items for the selected articles. A sub-sample of articles was checked for citation frequency to determine if a relationship existed between use of non-text items and levels of citation.

\section{DISCUSSION}

The research conducted used a large sample size, over a wide time span, and therefore the sample is likely to be representative of the population. More sophisticated statistical techniques would have been able to establish relevance and generalisability of the sample, however the resources to conduct this testing were not available. The sample selected for citation checking could have been broadened, with a larger sample over a wider time period possibly adding weight to the conclusion reached.

The implications of this research revolve around the norms relating to the use of non-text items. It would appear that articles that use higher levels of non-text items are more widely cited, so if citedness is the definition of 'success', then it may be prudent to utilise a wider range of non-text items.

The distribution of types of non-text items varied considerably between types of journals. Over $50 \%$ of the items observed in practitioner journals were graphics, whilst the major component of non-text items in academic journals was tables. The data derived during this research also contains information relating to individual journals preference for usage level and type of non-text items used. A taxonomy of journal preferences could be developed during future research; to increase the probability of publication in an author's preferred journal. Similar research by Vogel and Wetherbe (1984) examined journals to determine a taxonomy of preferred research methodologies, and universities to determine preferred journal for publication.

As an example of the differences observed between journals figure 9 shows results obtained when comparisons were drawn between types of non-text items used. 


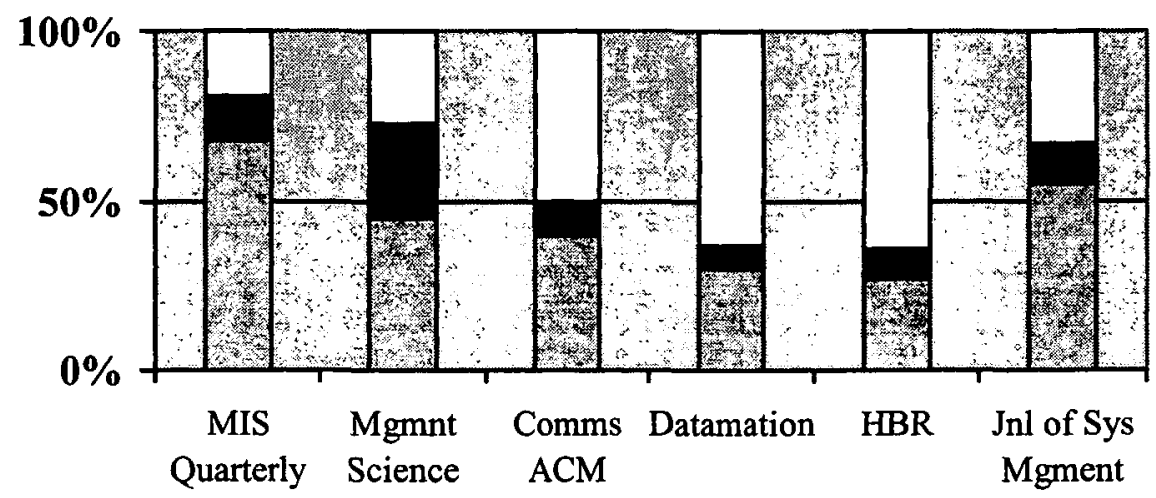

Tables Graphs DGraphics

Figure 9:Usage levels for different types of non-text items

An increasing use of colour was observed over time for the journals involved. 1970's non-text items tended to be black and white, and of relatively poor quality, whereas the 1980's and onwards showed a distinct improvement in the quality of non-text items used. Printing and editing technology has improved over the time of this analysis; increased complexity and colour was observed in articles that are more recent.

The type of graphics used has also varied considerably. 1970's magazines often used text items that were obviously 'cut and pasted' into the articles, apparent by the difference in font and general appearance to the flow of narrative. Later articles tended to use non-text items that blend with the narrative text, using similar font and colours to create a cohesive effect.

Articles in practitioner journals tended to be much shorter in length, on average 3 pages as opposed to an average 10 pages in academic journals. Additionally, practitioner journals contained articles that were more easily comprehended; however, conclusions were not necessarily supported by evidence. Additionally the referencing in practitioner articles tended to be sparse compared to the voluminous references included in academic articles.

If consideration is given to the motive behind selection of articles for citation when preparing research, there is a possibility that increased use of non-text items improves the clarity of research findings, leading to their increased usage, uptake and citation levels.

To summarise, consideration of a journal's preferred usage of non-text items might assist when submitting articles for publication. Use of a higher number of non-text items appears to lead to higher frequency of citation, and therefore increases the potential for recognition of the results of research endeavours.

\section{REFERENCES}

Benbasat I \& Dexter, A.S. (1986) "An Investigation of the Effectiveness of Colour and Graphical Information Presentation under Varying Time Constraints," MIS Quarterly, vol. 10, pp. $59-83$.

Davis, D. (1981) "An experimental investigation of the form of information presentation, psychological type of the user, and performance within the context." in College of Business. Florida: University of Florida.

Hamilton S \& Ives, B. (1983) "The Journal Communication System for MIS Research," Data Base, vol. Winter, pp. 3 - 14.

Owen, J Mackenzie \& van Halm, J. (1989) Innovation in the information chain, 1st ed. London: Routledge.

McGill, L.T. (1990) "Doing Science by the Numbers," in The Rhetoric of Social Research: Understood and Believed, A. Hunter, Ed. New Brunswick: Rutgers U.P.

Moran, M. \& Journet, D. (1985) "Research in Technical Communication," 1st ed. Connecticut: Greenwood Press.

Vogel, D.R. \& Wetherbe, J.C. (1984) "MIS Research: A profile of leading Journals and Universities," Data Base, vol. Fall, pp. 3 - 14. 